University of Nebraska - Lincoln

DigitalCommons@University of Nebraska - Lincoln

$6-2005$

\title{
Transitioning from analog to digital audio recording in childhood speech sound disorders
}

\author{
Lawrence D. Shriberg \\ University of Wisconsin-Madison, shriberg@waisman.wisc.edu \\ Jane L. McSweeny \\ University of Wisconsin-Madison, \\ Bruce E. Anderson \\ University of Wisconsin-Madison,, anderson@waisman.wisc.edu \\ Thomas F. Campbell \\ University of Pittsburgh, tfc061000@utdallas.edu \\ Michael R. Chial \\ University of Wisconsin-Madison \\ See next page for additional authors
}

Follow this and additional works at: https://digitalcommons.unl.edu/specedfacpub

Part of the Special Education and Teaching Commons

Shriberg, Lawrence D.; McSweeny, Jane L.; Anderson, Bruce E.; Campbell, Thomas F.; Chial, Michael R.; Green, Jordan R.; Hauner, Katherina K.; Moore, Christopher A.; Rusiewicz, Heather L.; and Wilson, David L., "Transitioning from analog to digital audio recording in childhood speech sound disorders" (2005). Special Education and Communication Disorders Faculty Publications. 58.

https://digitalcommons.unl.edu/specedfacpub/58

This Article is brought to you for free and open access by the Department of Special Education and Communication Disorders at DigitalCommons@University of Nebraska - Lincoln. It has been accepted for inclusion in Special Education and Communication Disorders Faculty Publications by an authorized administrator of DigitalCommons@University of Nebraska - Lincoln. 


\section{Authors}

Lawrence D. Shriberg, Jane L. McSweeny, Bruce E. Anderson, Thomas F. Campbell, Michael R. Chial, Jordan R. Green, Katherina K. Hauner, Christopher A. Moore, Heather L. Rusiewicz, and David L. Wilson 
Published in Clinical Linguistics \& Phonetics 19:4 (June 2005), pp.: 335-359; doi:

10.1080/02699200500085426 Copyright @ 2005 Taylor \& Francis Ltd. Used by permission.

Submitted January 27, 2004; accepted March 22, 2004.

\title{
Transitioning from analog to digital audio recording in childhood speech sound disorders
}

\author{
Lawrence D. Shriberg, ${ }^{1}$ Jane L. McSweeny, ${ }^{1}$ Bruce E. Anderson, ${ }^{1}$ \\ Thomas F. Campbell, ${ }^{2}$ Michael R. Chial, ${ }^{1}$ Jordan R. Green, ${ }^{3}$ \\ Katherina K. Hauner, ${ }^{1}$ Christopher A. Moore, ${ }^{4}$ \\ Heather L. Rusiewicz, ${ }^{2}$ and David L. Wilson ${ }^{1}$ \\ 1. University of Wisconsin-Madison, WI, USA \\ 2. University of Pittsburgh, Pittsburgh, PA, USA \\ 3. University of Nebraska-Omaha, Omaha, NE, USA \\ 4. University of Washington, Seattle, WA, USA \\ Corresponding author - Lawrence D. Shriberg, Phonology Project, Waisman Center, \\ University of Wisconsin-Madison, 1500 Highland Avenue, Madison, WI 53705, USA; \\ e-mail shriberg@waisman.wisc.edu
}

\begin{abstract}
Few empirical findings or technical guidelines are available on the current transition from analog to digital audio recording in childhood speech sound disorders. Of particular concern in the present context was whether a transition from analog- to digital-based transcription and coding of prosody and voice features might require re-standardizing a reference database for research in childhood speech sound disorders. Two research transcribers with different levels of experience glossed, transcribed, and prosody-voice coded conversational speech samples from eight children with mild to severe speech disorders of unknown origin. The samples were recorded, stored, and played back using representative analog and digital audio systems. Effect sizes calculated for an array of analog versus digital comparisons ranged from negligible to medium, with a trend for participants' speech competency scores to be slightly lower for samples obtained and transcribed using the digital system. We discuss the implications of these and other findings for research and clinical practice.
\end{abstract}

Keywords: articulation, assessment, phonology, prosody, transcription

\section{Introduction}

As is widely occurring among professionals in such disciplines as medicine, law, and business informatics, researchers and clinicians in communicative disorders are transitioning from analog to digital methods to record, store, and play back audio and video information. A brief sample of the wide-ranging sources of information relevant to the transition to digital methods for audio recording in communicative disorders includes early and more recent discussions of the advantages and disadvantages of digital recording and signal processing (e.g., Titze, Horii, \& Scherer, 1987; Doherty \& Shipp, 1988; Perry, Ingrisano, \& Blair, 1996; Farinella, DeLeo, Metz, Schiavetti, \& Whitehead, 1998; Evans \& Miller, 1999; Zafar, Overhage, \& McDonald, 1999; Bunta, Ingram, \& In- 
gram, 2003), database storage and access issues (e.g., Bamattre, 1995; Long, 1999; Beukelman, 2000; Bunta et al., 2003; MacWhinney, 2005), and training suggestions for digitally based phonetic transcription and other acoustic-perceptual tasks (Wolfe, Martin, Borton, \& Youngblood, 2003). Except for the useful tutorial by Bunta, Ingram, and Ingram (2003) as well as the more general guidelines in Plichta (2002) and Ladefoged (2003), a literature review yielded few sources specifically focused on the topic of interest-the assembly of digital systems for glossing, phonetic transcription, and prosody-voice coding of samples from children with speech sound disorders. The following sections discuss two types of information needed by researchers and clinicians transitioning to digital audio systems for these purposes.

\section{Psychometric issues associated with digital audio systems}

A primary need in transitioning from analog to digital audio media is for a quantitative comparison of findings obtained with digital systems to extant archival data obtained with analog systems. As in most other areas of communicative disorders, the reference standards that continue to be used to describe and classify typical and atypical speech acquisition were obtained using analog recording and playback media. The reliability and validity of these audio-recorded data were assessed in a number of early methodological studies comparing transcription and scoring of live speech to that of recorded samples (see reviews in Shriberg \& Lof, 1991; Shriberg \& Kent, 2003). Findings from these studies generally supported the validity and reliability of transcription from audio-taped recordings. Surprisingly, however, there is no body of clinical-research studies that has compared transcription from earlier reel-to-reel recorders with transcription from later and current generations of audiocassette recorders. Notwithstanding certain attractive efficiency features of some of the more widely used (but lower-end )audio cassette recorders, technical limitations in characteristics such as bandwidth, signal-tonoise ratio, and dynamic range have yielded recordings of generally poorer quality than those produced with an earlier generation of higher-end reel-to-reel recorders.

\section{Configuring a digital audio system for speech sampling}

A second and related need on this topic in the laboratory and clinic is for guidance in selecting the appropriate format and specific hardware and software to configure a digital audio system to record, store, and play back speech samples. During the period when dedicated analog devices were the only available choice for audio recording, purchasing decisions were primarily based on the answers to three questions: which type, brand, and model of reel-to-reel or audiocassette recorder is technically appropriate and financially feasible for the intended speech sampling tasks (e.g., sound quality, portability, reliability, ease of use, purchase price, maintenance costs)? Which microphone best meets the technical and substantive requirements of one or more speech sampling tasks (e.g., sensitivity, frequency response, directionality, signal-to-noise ratio)? Which type of audio tape is most suitable for recording and storage needs (e.g., frequency response and bias, resistance to stretching, playing time)? For playback purposes, the primary consideration was to choose a device, including matched earphones and/or loudspeaker(s), thatwas technically adequate and efficient for the repeated playbacks needed for auditoryperceptual tasks (e.g., glossing, transcribing, scoring real and nonsense words, scoring diadochokinetic speech tasks, rating intelligibility, coding prosody-voice features). 
In contrast to analog technology, contemporary digital formats for audio speech sampling include a large array of recording-storage-playback options, generally based on recording and/or storage medium needs (e.g., digital audio tape [DAT], compact discs [CD], minidiscs [MD], digital versatile discs [DVD]). Selecting, purchasing, assembling, and maintaining the several components of a digital audio system, particularly as they interface with desktop or notebook computers, are typically guided by consultation with experienced colleagues or local audio vendors. Such sources of guidance may not always be effective for persons responsible for one or multiple digital systems for use in academic, research, or clinical settings. Colleagues may not be acquainted with the range of available technical and vendor options; technical consultants or vendors may not be acquainted with the requirements and specific constraints associated with recording, glossing, and transcribing the speech of young children with speech sound disorders.

\section{Statement of the problem}

The present report describes an analog-to-digital transition experience in a clinicalresearch context. The general need was to configure multiple, laptop-based playback stations for use by research transcribers to reduce auditory-perceptual data from the digital recordings of assessment protocols administered to typical and atypical speakers across the life span. The primary focus of the present study was on conversational speech sampling from young children with moderate to severe speech disorders. However, the system was also intended to be used for other assessment tasks, such as citation-form articulation tests, word-phrase and sentence-length imitation tasks, and intelligibility assessment. The research design addressed the following question: do data obtained using a representative digital audio system differ significantly from data obtained using a representative analog audio system? As indicated in the discussion above, the primary concern was whether a transition from analog- to digitally-based transcription and prosody-voice coding would require restandardizing a reference database.

\section{Method}

\section{Participants}

Eight conversational speech samples were selected from a set of 35 samples from children whose speech had been assessed at the Children's Hospital of Pittsburgh. These speakers, each of whom was referred by a certified speech-language pathologist as having a speech production disorder, were participants in an ongoing collaborative study of physiological correlates of child speech sound disorders. Table I includes descriptive information for the eight participants with speech delay whose conversational speech samples were glossed, transcribed, and prosody-voice coded for the present study.

As shown in Table I, the eight speakers ranged in age from 3 years, 2 months to 4 years, 6 months (mean age: 4 years, 2 months). This average age is comparable to the mean age of 4 years, 3 months reported in a study profiling several hundred children with speech delay referred to a local university speech clinic (Shriberg \& Kwiatkowski, 1994). Seven of the eight (88\%) children were males, which is slightly higher than the distribution of 70\% males reported in Shriberg and Kwiatkowski (1994). As shown in Table I, five of the eight (63\%) children sampled for the present study had Moderate-to-Se- 
Table I. Information on the eight participants with speech delay and the transcribers' schedule for completing auditory-perceptual tasks using analog and digital audio systems.

\begin{tabular}{|c|c|c|c|c|c|c|c|c|}
\hline \multirow{3}{*}{$\begin{array}{l}\text { Participant } \\
\text { Number }\end{array}$} & \multirow{3}{*}{$\begin{array}{c}\text { Age } \\
\text { (mos.) }\end{array}$} & \multirow[b]{3}{*}{ Sex } & \multirow{3}{*}{$\begin{array}{l}\text { Estimated Speech } \\
\text { Severity }\end{array}$} & \multicolumn{4}{|c|}{ Transcription Schedule } & \multirow{3}{*}{$\begin{array}{l}\text { Length of } \\
\text { Conversational } \\
\text { Sample (min:sec) }\end{array}$} \\
\hline & & & & \multicolumn{2}{|c|}{ Transcriber 1} & \multicolumn{2}{|c|}{ Transcriber 2} & \\
\hline & & & & Time 1 & Time 2 & Time 1 & Time 2 & \\
\hline 1 & 38 & M & Mild & $A^{a}$ & $\mathrm{D}$ & $\mathrm{D}$ & $A$ & $7: 28$ \\
\hline 2 & 42 & M & Moderate-Severe & A & $\mathrm{D}$ & $\mathrm{D}$ & $A$ & $16: 52$ \\
\hline 3 & 47 & M & Moderate-Severe & $\mathrm{D}$ & $A$ & - & - & $10: 40$ \\
\hline 4 & 49 & $\mathrm{~F}$ & Moderate-Severe & $A$ & D & $A$ & $\mathrm{D}$ & $11: 58$ \\
\hline 5 & 51 & $M$ & Mild & $\mathrm{D}$ & $A$ & - & - & $10: 47$ \\
\hline 6 & 51 & M & Mild & $\mathrm{D}$ & $A$ & - & - & $7: 18$ \\
\hline 7 & 53 & M & Moderate-Severe & $\mathrm{D}$ & $A$ & - & - & $12: 24$ \\
\hline 8 & 54 & M & Moderate-Severe & $A$ & D & $A$ & $\mathrm{D}$ & $5: 43$ \\
\hline
\end{tabular}

a. $A=$ Analog; $D=$ Digital

vere speech involvement. Quantitative speech metrics to be reported as part of the dependent variables under study supported these a priori clinical impressions of speech severity.

\section{Research design}

The independent variable of primary interest was type of audio system (analog versus digital), with secondary interest in possible effects associated with the experience level of the transcriber. Table 1 includes the schedule for transcription and prosody-voice coding of the speech samples, as completed by two research transcribers whose backgrounds will be described. Two series of listening sessions, each completed within a 3- to 10-day period, were scheduled independently for each transcriber, with the second series (Time 2) having begun 1 month after the first series (Time 1). Transcriber 1 glossed, transcribed, and prosody-voice coded all eight of the speech samples, using the counterbalanced schedule of analog (A) and digital (D) playback systems shown in Table I. Transcriber 2 followed the same procedure for four of the eight samples. Three of Transcriber 2's four samples (75\%) were randomly selected from participants whose severity of speech involvement was judged to be Moderate-Severe. Transcriber 1's first and last glossed utterances in the first listening series were used as starting and ending reference points to derive playing time lengths for each conversational speech sample. As shown in Table I, the durations of the speech sample sections (including examiner utterances) eventually used for transcription and prosody-voice coding ranged from $5 \mathrm{~min}$ $43 \mathrm{~s}$ to $16 \min 52 \mathrm{~s}(\mathrm{M}=10 \min 24 \mathrm{~s}, \mathrm{SD}=3 \mathrm{~min} 34 \mathrm{~s})$.

\section{Speech samples}

A conversational speech sample was acquired from each participant using both the analog and digital recording media described in the Appendix. The conversational speech task was collected on the first day of the 2-day assessment protocol, during a point when participants were familiar and comfortable with the examiner and task expectations. The children were tested by one of four trained examiners (certified speech-language pathologists), who were experienced in evoking spontaneous speech from young children. A set of examiner guidelines was followed, which included instructions for obtaining linguisti- 
cally rich conversational samples, for monitoring the recording level to maximize signal quality, and for glossing of strings of questionably intelligible speech. Concerning the latter need, the examiner would gloss what she perceived as the child's intended linguistic targets, repeating the utterance approximately word-for-word on the audio recording.

\section{Analog and digital audio systems}

The Appendix provides technical information on the analog and digital audio systems used to record and play back the speech samples. Essentially, the conversational speech samples in the parent study were recorded with a common audio signal routed to an audiocassette recorder for the analog samples, and a digital audio tape recorder for the digital samples. As described in the Appendix, the playback component of the analog system was a foot-pedal operated device used in prior research in child speech sound disorders. The playback software for the digital system running on a personal computer was a foot-pedal operated media player developed specifically for this project.

\section{Phonetic transcription and prosody-voice coding}

Training. Transcriber 1 (author McSweeny) had 10 years' experience in obtaining, glossing, transcribing, and prosody-voice coding speech samples from children with speech disorders of known and unknown origin. Transcriber 2, an undergraduate student in Communicative Disorders, had 4 months' experience in glossing, transcribing, and prosody-voice coding conversational samples. This 4-month period included a 2.5-month training program conducted by Transcriber 1 and included both knowledge components and auditory-perceptual skills training. Knowledge-based training consisted of directed reading assignments in articulatory phonetics that provided (a) rules for use of the 42 phonetic and 43 diacritic characters included in the system for narrow phonetic transcription described in Shriberg and Kent (2003), (b) rules for the use of 31 exclusionary and 32 prosody-voice codes included in the system for prosody-voice coding described in Shriberg, Kwiatkowski, and Rasmussen (1990), and (c) rules and guidelines for the array of glossing and formatting principles included in a software program described originally in Shriberg (1986) and updated in Shriberg, Allen, McSweeny, and Wilson (2001). Skills-based training included the completion of all auditory modules in the previous references, additional training on samples of children with mild to severely delayed speech, and corrective feedback based on assessments of interjudge agreement with Transcriber 1. All training was conducted using an analog playback device similar to the one used in the present study. Brief training was also provided in use of the digital playback software.

Procedure. Glossing, phonetic transcription, and prosody-voice coding of the conversational speech samples were completed in a small listening room. The analog playback system and the tabletop computer for the digital system were positioned on the same work surface, with their corresponding foot pedals also placed adjacently. The order of the conversational samples in both listening series followed the counterbalanced analog-digital design shown in Table I. During and immediately after each session, the transcribers used worksheets both to log elapsed times for each auditory perceptual task and to annotate their observations about the operational features of each audio system, including any personal preferences. 
Analyses. Transcribers' glosses, phonetic transcriptions, and prosody-voice codes were first checked for formatting accuracy and then were entered into a software suite using editing utilities (Shriberg et al., 2001). The software included outputs that provided detailed quantitative information for all study questions.

Effect sizes and their associated 95\% confidence intervals, using pooled standard deviations and Hedges' correction (Hedges \& Olkin, 1985; Cohen, 1988), were selected from the statistical options in the analysis software. These metrics estimated the clinical research significance of any obtained differences in the completion times, reliability, and validity comparisons of data obtained using the analog versus digital audio systems. Cohen's (1988) traditional descriptor system and magnitude criteria were used to characterize three effect size (ES) ranges as follows: $\leq .19=$ negligible, $.20-.49=$ small, .50-.79 $=$ medium. To provide a means to differentiate among effect sizes greater than Cohen's largest ES descriptor (i.e., > .79 = large), such values were redefined as large (.80-.99), very large (1.0-1.99), and extremely large ( $\geq 2.0$; see Hopkins, 2003 , for a discussion of rationale for these distinctions). Following the usual convention, 95\% ES confidence intervals not containing 0 were considered statistically significant. To aid the reader in examination of the several data tables, however, we also provide the equivalent inferential statistical findings from two-sample, pooled variance $t$ tests. Given the goals of this initial study, and its limitations due to small cell sizes, the inferential statistical findings were deemed only advisory. Rather, any pattern of comparisons yielding medium and especially large effect sizes was of primary interest for issues associated with standardization and other issues in clinical-research applications of digital audio systems.

\section{Results and discussion}

\section{Glossing}

The first auditory-perceptual task for each speech sample was to gloss the sample until 100 different word types (i.e., unique lexical entries termed first occurrence words: cf. Shriberg \& Kwiatkowski, 1980) had been glossed. The speech samples from Participants 2 and 4, whose speech was considerably unintelligible, yielded only 79 and 83 first occurrence words, respectively, but were included in the analyses nevertheless. To allow estimates of intrajudge and interjudge agreement for all three auditory-perceptual tasks using the two audio systems, Transcriber 2 first glossed each sample but subsequently used Transcriber 1's glosses for transcription and prosody-voice coding. Thus, Transcriber 2's glosses provided the information from which intrajudge glossing agreement percentages were obtained, but her use of Transcriber 1's gloss for transcription and prosody-voice coding data allowed the agreement software to calculate intrajudge and interjudge agreement percentages for these tasks based on similar presumed linguistic targets.

\section{Completion time}

Findings. Table II includes completion time data for glossing completed by Transcriber 1 (eight samples) and Transcriber 2 (four samples), at the first (Time 1) and second (Time 2) listening series using the two audio systems (analog, digital). The completion time data for glossing in Table II are longer than would normally occur for transcription alone, because they included the time needed to indicate utterances that had to be ex- 


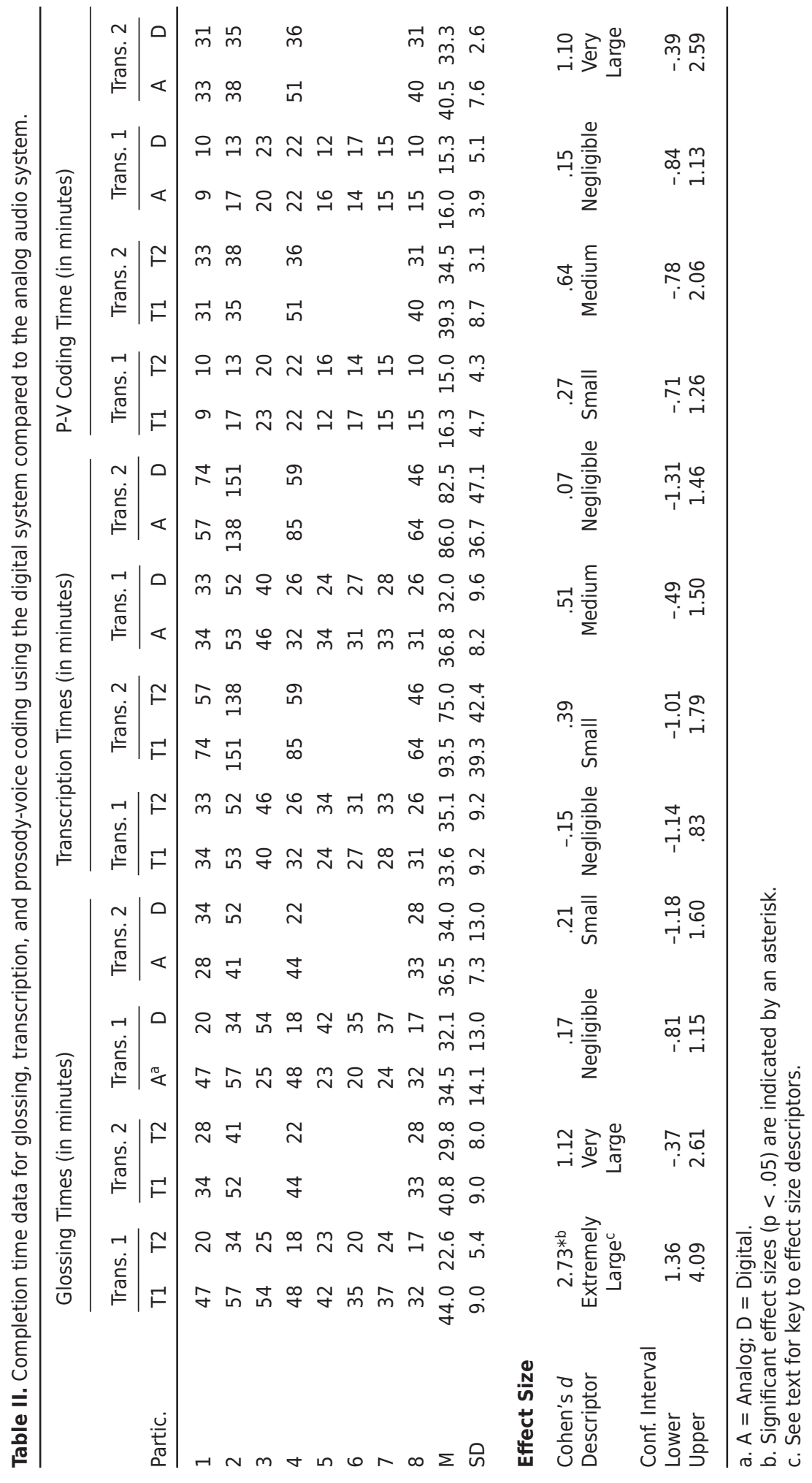


cluded from prosody-voice coding (termed exclusion coding: cf. Shriberg et al., 1990), due to technical and/or linguistic confounds.

As shown in Table II, glossing times (in minutes) for both transcribers were considerably reduced for the second listening series period relative to the first series. The extremely large (2.73) ES (effect size) for Transcriber 1 was statistically significant, with the completion time for glossing the eight speech samples reduced by approximately $50 \%$ from the first $(M=44.0 \mathrm{~min})$ to the second $(M=22.6 \mathrm{~min})$ listening series. The very large ES (1.12) for Transcriber 2 (who glossed only four samples) was not statistically significant, with samples reduced by approximately $25 \%$ from the first $(\mathrm{M}=40.8$ $\mathrm{min})$ to the second $(\mathrm{M}=29.8 \mathrm{~min}$ ) listening series. Transcriber 1 averaged $34.5 \mathrm{~min}$ and 32.1 min using the analog and digital systems, respectively; Transcriber 2 averaged $36.5 \mathrm{~min}$ and $34.0 \mathrm{~min}$ for each system, respectively. As shown in Table II, the ESs associated with these approximately 4-9\% reductions in time from analog to digital for both transcribers were negligible and small, respectively, and not statistically significant.

Discussion. The anecdotal logs from the transcribers indicated that the statistically significant reductions in gloss times in the second series, regardless of which audio system was used (see individual comparisons in Table II), were due to a memory confound. Although both transcribers had transcribed many other samples since the first listening series one month previously, Transcriber 1 reported that she remembered some conversational content from the first series, and Transcriber 2 reported that she remembered a great deal of the content from the first series of samples. The recalled information aided in reducing the number of playbacks needed to identify and recheck glosses of initially unintelligible words. Notwithstanding this methodological confound, the transcribers' glossing times were not significantly different using the digital system compared to the analog audio system.

\section{Reliability}

Between-session agreement. The glossing agreement data in the left section of Table III begin with reliability information on the total number of utterances and words glossed by each transcriber in each session (first two row variables), including means and standard deviations for the per-sample occurrence rates. As indicated by the negligible ES values for all four comparisons, the total number of utterances and words glossed was considered comparable in the two listening sessions.

The remaining four rows in Table III provide the percentage of glossing agreements, Time 1/Time 2 totals, and per-sample average values (means, standard deviations) for four classes of glossed words: words a transcriber could readily gloss (non-questionables), non-questionable words that by rule were excluded from the speech analyses (disregards; e.g., multiple sequential repetitions of a word), words a transcriber could gloss with some difficulty ( questionables), and words a transcriber could not gloss (unintelligibles). As shown in Table III, the between-session percentages of exact agreement for these four glossing categories across the two audio systems ranged widely from $21.5 \%$ to $84.8 \%$ point-to-point agreement (excluding the low occurrence of questionables for Transcriber 2). However, associated ESs for differences in the total occurrence of each word class in the two sessions ranged from .02 to .57, with only the ES associated with disregards for Transcriber 1 ( $E S=.57)$ reaching the criterion for a medium difference. 
Between-system agreement. The primary reliability analysis (right section of Table III) yielded comparable numbers of utterances and words for each transcriber's gloss using the digital compared to the analog system (ESs from -.01 to -.08) and comparable numbers for each of the four word classes (ESs from .07 to -.34). Four of the latter seven computed ESs were classified as negligible to small and were not statistically significant. Point-to-point exact percentages of agreement between transcribers within each audio system ranged from $19.4 \%$ to $88.4 \%$ for the four types of glossed words.

Discussion. The low levels of exact point-to-point intrajudge (between-session) and interjudge (between-system) agreement for some of the four word classes are consistent with difficulties in glossing the speech of children with moderate to severe intelligibility problems (Shriberg \& Lof, 1991; Weston \& Shriberg, 1992). Both transcribers noted that most of these samples were among the most challenging they had ever been assigned to gloss and transcribe. Additional discussion of factors and processes underlying examiners' and transcribers' "guesses" at children's intended word forms goes beyond the scope of the present paper. From the present methodological perspective, it is important to note that some types of between-class differences in glossing have greater consequences than others for validity issues (e.g., for classification of children's speech status, or for treatment recommendations). In the present context, the most important word class data are the values for the non-questionable words. As shown in Table III, the two audio systems have comparable total numbers of non-questionable words, comparable percentages of nonquestionable words in the sample, and a relatively high percentage of exact agreement for non-questionable words (ranging from $79.3 \%$ to $88.4 \%$ across the four estimates). The glossing agreement data for each of the word classes in Table III are interpreted as indicating that auditory-perceptual judgments based on the signal provided by the analog system were comparable to those based on the signal provided by the digital system.

\section{Phonetic transcription}

Completion time analyses. Findings. Table II includes completion time data for narrow phonetic transcription of the eight and four samples transcribed by Transcriber 1 and Transcriber 2, respectively, for each of the two listening series and two audio systems. ES estimates for the four listening series comparisons ranged from negligible to medium, with none statistically significant. As shown in both the per-sample and summary data for each listening series, there were substantial differences in the lengths of time needed by each transcriber to complete transcription. Transcriber 2 averaged $93.5 \mathrm{~min}$ and $75.0 \mathrm{~min}$ for the four samples she transcribed in the first and second listening series respectively, whereas Transcriber 1 averaged only $33.6 \mathrm{~min}$ and $35.1 \mathrm{~min}$ for the eight samples she transcribed in the first and second listening series. Comparable completion time differences were obtained for the four samples transcribed by both transcribers.

Discussion. As with the completion time findings for glossing (Table II), the completion time data for phonetic transcription indicated that there were no significant time differences associated with the digital versus the analog system. Unlike the findings for the glossing data, however, which indicated that the two transcribers took about the same length of time to gloss the four samples, the inexperienced transcriber (Transcriber 2) took considerably more time than the experienced transcriber to phonetically transcribe the samples using either audio system. As shown in Table II, Transcriber 2 averaged 


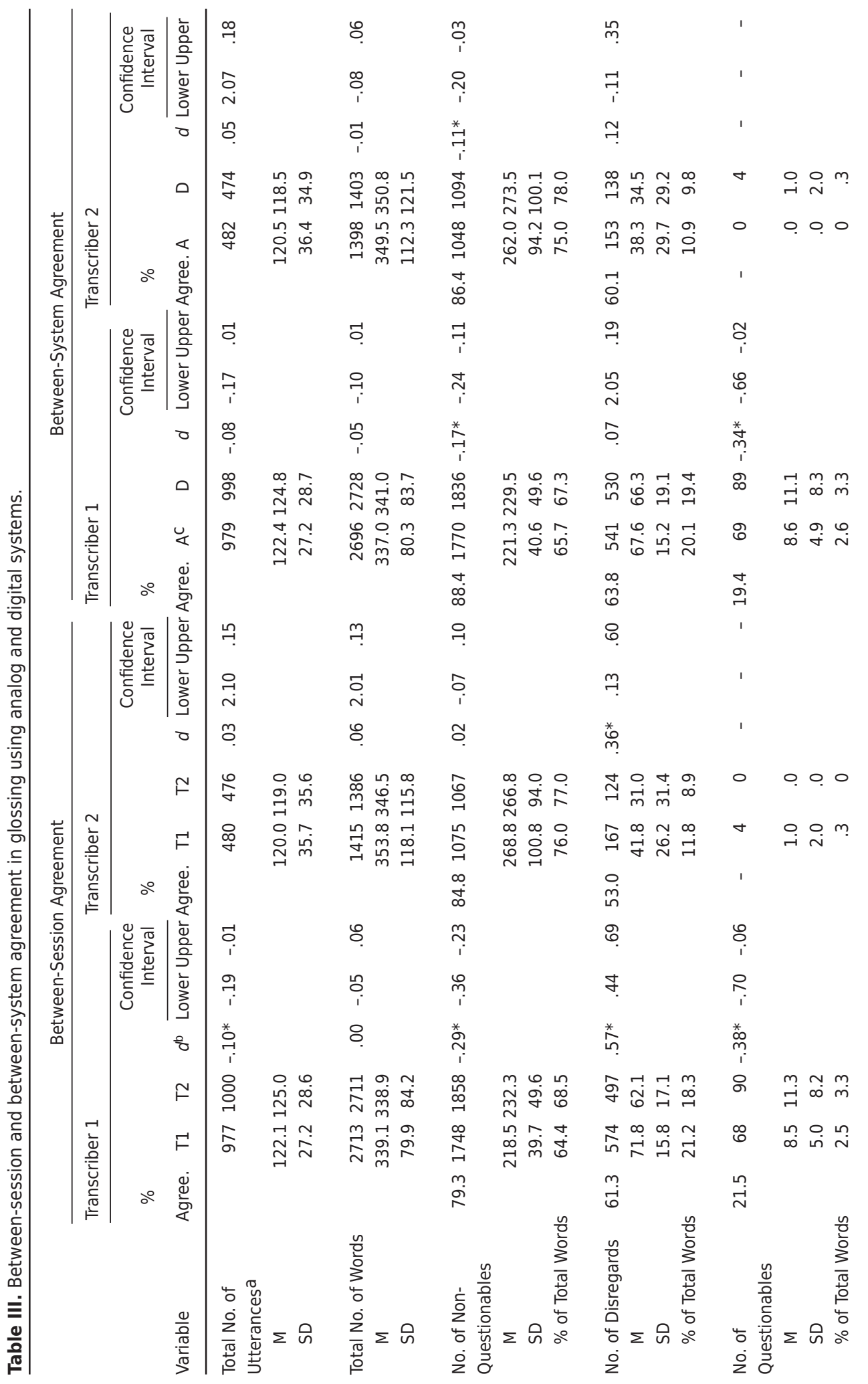




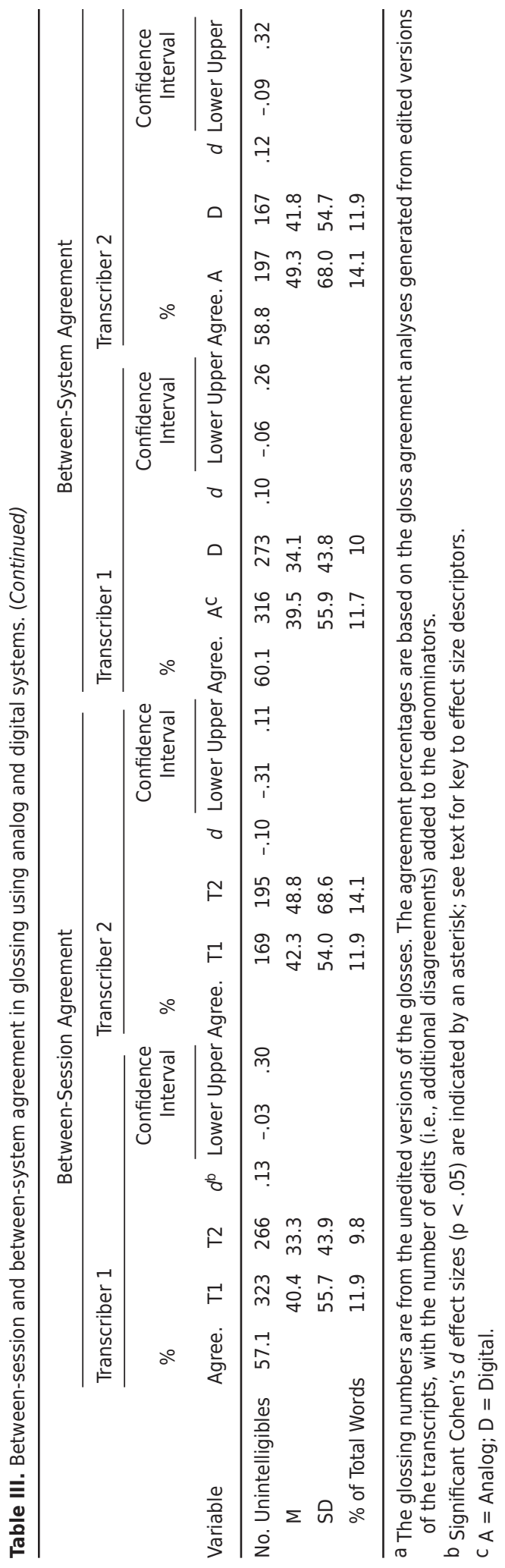


approximately one and one-half hours per sample to complete a phonetic transcription in the first listening series, approximately two to three times longer than Transcriber 1's average of just over one-half hour per sample for the first listening series. These differences for both audio systems were evidently due to the additional time needed for Transcriber 2 to make perceptual-cognitive decisions, including more playbacks as well as longer deliberations about narrow phonetic transcription conventions and the use of alternative diacritics to capture auditory percepts. Thus, the primary efficiency finding is that, compared to the analog system, the presumably clearer signal and more efficient playback available in the digital system (see later discussion) were not associated with reduced completion times for narrow phonetic transcription for either the experienced or the relatively inexperienced transcriber.

\section{Reliability}

Table IV is a summary of the point-to-point between-system and between-transcriber transcription agreement findings. As shown in the first four rows, between-system (intrajudge) comparisons were based on 977 utterances (1690 words used) for the eight speech samples transcribed by Transcriber 1, and 484 utterances (833 words used) for the subset of four speech samples transcribed by Transcriber 2 . Because both transcribers transcribed each speech sample only once using each audio system, it was not possible to estimate intrajudge agreement within each system.

Between-system findings and discussion. Point-to-point agreement estimates were obtained for broad and narrow phonetic transcription of vowels and consonants. The eight between-system percentage of agreement estimates for the two transcribers ranged from $83.4 \%$ to $94.9 \%$. Within these relatively high levels of agreement for transcription using the two audio systems, Transcriber 2's average levels of agreement were substantially higher, as indicated by the magnitudes of both the agreement percentages and the large to extremely large ES descriptors (two of which were statistically significant). As with most of the present data based on relatively few samples, these ES estimates were bounded by wide confidence intervals.

The increased between-session agreement for Transcriber 2 compared to Transcriber 1 may be explained by the associated completion time data (Table II) and the anecdotal comments reported previously. As described previously, Transcriber 2 took two to three times longer than Transcriber 1 to transcribe the samples. She also reported that in the second listening series she was able to recall much of the content as well as her transcription of the speech samples heard in the first listening series. Transcriber 1, who had been involved in many more research tasks during the 1-month interval between sessions, reported more limited recall of the speech sample content. Thus, the timetable for the study and the decision not to limit the maximum number of allowable replays (training guidelines suggested a maximum of three replays but permitted as many as needed) were likely sources contributing to the differences in the between-session agreement percentages for the two transcribers.

Between-transcriber findings and discussion. The remaining data in Table IV reflect transcriber agreement for the four samples completed within each of the two audio systems. Interjudge agreement percentages ranged from 69.1\% (analog system: narrow transcription of consonants) to $86.5 \%$ (analog system: broad transcription of vowels). The ES esti- 


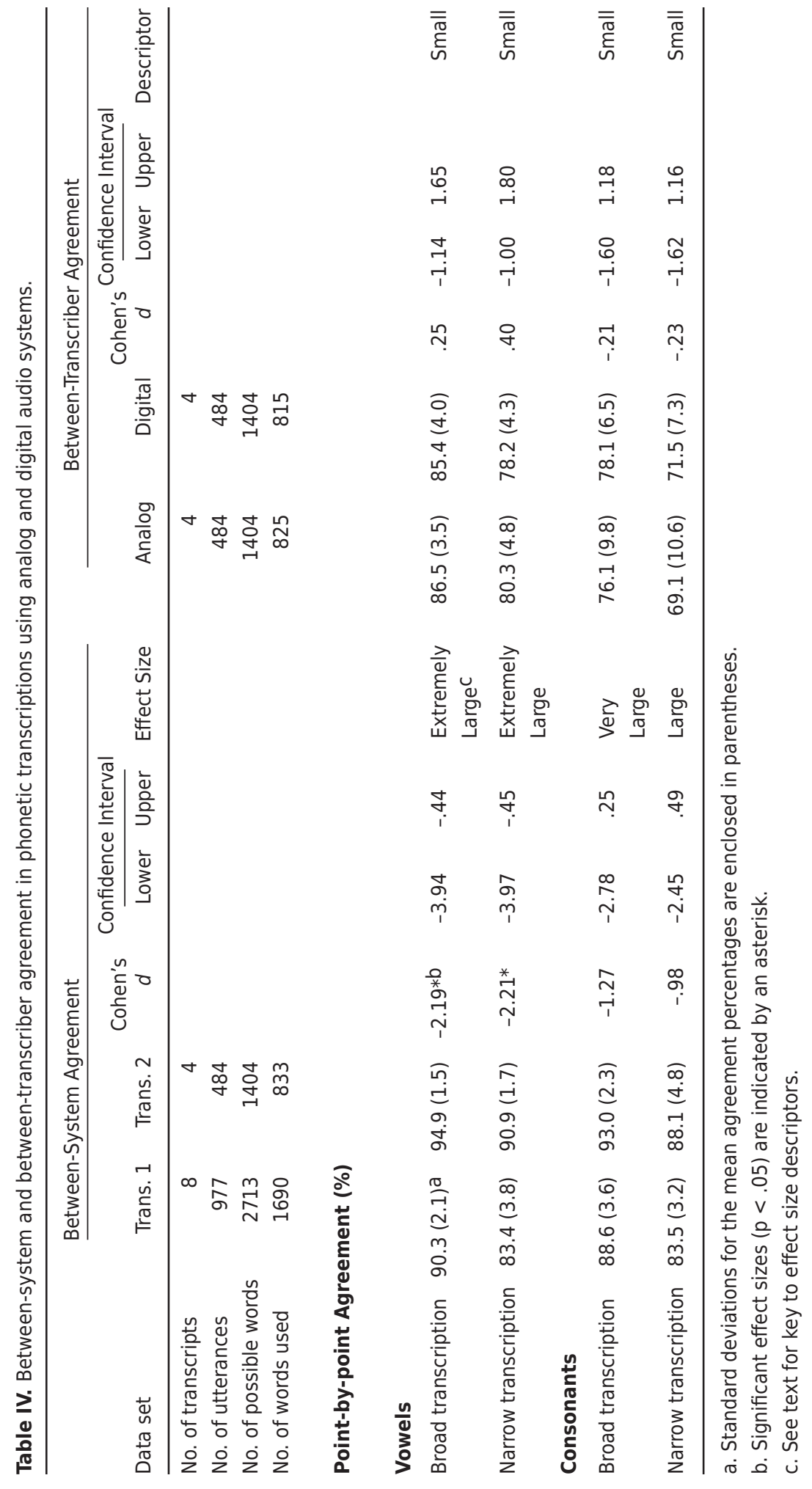


mates computed for these interjudge agreement comparisons within each audio system were small, with none meeting criteria for statistical significance. Thus, for the total of four speech samples, transcribers' interjudge agreement was not significantly higher for transcription completed with the digital system compared to the analog audio system.

In addition to the summary statistics shown in Table IV, the agreement software provided detailed information aggregated by place-manner features, phonemes, and diacritics. These outputs were examined in an attempt to identify the primary sources of disagreements in the summary transcription agreement findings reported above. Table $\mathrm{V}$ is a summary of findings, organized by target consonant or rhotic vowel sound (top section) and target manner feature (bottom section). The between-system (i.e., analog, digital) agreement percentages for each set of comparisons are rank-ordered from highest to lowest, based on the mean narrow transcription percentages from both transcribers (calculated as the sum of the means for each transcriber, divided by two). Thus, for example, the $/ 3 y$ phoneme was ranked as the most challenging sound to transcribe reliably (ranked 25th), based on the average percentage of analog-digital agreement for the two transcribers using narrow phonetic transcription (61.2\%).

A methodological constraint on the interpretation of data in Table V warrants comment. The number of occurrences of the 23 target consonants and two target rhotics in the eight samples transcribed by Transcriber 1 ranged from three to 285 tokens. A comparably wide range of target phoneme tokens (1-157) occurred within the four conversational speech samples transcribed by Transcriber 2. In addition to the low distributional rates of occurrence in conversational speech for certain phonemes (cf. Shriberg \& Kent, 2003: Appendix B), consonants most likely to be misarticulated may have had low target occurrence rates in certain transcripts due to intelligibility constraints and possibly to speaker avoidance constraints. Thus, interpretation of the summary and especially the per-phoneme transcription agreement figures for analog versus digital audio systems is constrained by the number of occurrences for which such reliability estimates were computed. Notably, for example, interpretation of the agreement percentages for the highest (/dz/) and lowest (/S/, $/ 3^{2} /$ ) ranked phonemes in Table $\mathrm{V}$ should be tempered by this constraint.

All of the summary (Table IV) and phoneme and feature (Table V) transcription agreement percentages for analog-digital system comparisons are within the ranges of pointto-point agreement estimates reported for analog-based transcription of children with mild to severe speech disorders of unknown origin (cf. Shriberg \& Lof, 1991; McSweeny \& Shriberg, 1995). Specifically, narrow transcription agreement percentages for a small group of phonemes are often no higher than the mid- to high-60s, especially when transcribing the most challenging speech samples, using a large array of diacritic symbols to describe error and non-error allophones. Thus, keeping in mind the two methodological constraints noted above, the audio signal available in the digital system was not associated with greater interjudge agreement. As noted earlier, intrajudge agreement using each of the audio systems was not estimated in the present design. However, it is clear that alternative diacritic description of speech (i.e., narrow phonetic transcription) was the major source of variance within each of the two audio systems. Three aspects of the data support this interpretation.

First, examination of diacritic summaries indicated that both transcribers used the typically large number of diacritics needed to describe the clinical and non-clinical distortions that occur in children with moderate to severe speech delay (cf. Shriberg, 1993: Appendix). For the four samples transcribed by both transcribers, Transcriber 1 used 
Table V. Rank-ordered disagreement findings for transcription completed using the two audio systems. Rank ordering is based on the mean agreement for both transcribers for narrow transcription.

\begin{tabular}{|c|c|c|c|c|c|c|c|c|c|}
\hline \multicolumn{10}{|c|}{ Rank Ordered Agreement by Target Consonant Sounda } \\
\hline \multirow[t]{2}{*}{ Rank } & \multirow[t]{2}{*}{$\begin{array}{l}\text { Target } \\
\text { Sound }\end{array}$} & \multicolumn{2}{|c|}{$\begin{array}{l}\text { Mean of Both } \\
\text { Transcribers }\end{array}$} & \multicolumn{3}{|c|}{ Transcriber 1 (eight samples) } & \multicolumn{3}{|c|}{ Transcriber 2 (four samples) } \\
\hline & & Broad & Narrow & $\mathrm{n}$ & Broad & Narrow & $\mathrm{n}$ & Broad & Narrow \\
\hline 1 & $/ d 3 /$ & 100.0 & 100.0 & 3 & 100.0 & 100.0 & 2 & 100.0 & 100.0 \\
\hline 2 & $/ \mathrm{W} /$ & 100.0 & 98.7 & 113 & 100.0 & 97.3 & 48 & 100.0 & 100.0 \\
\hline 3 & $/ \mathrm{h} /$ & 99.3 & 98.6 & 136 & 98.5 & 97.1 & 72 & 100.0 & 100.0 \\
\hline 4 & $/ \mathrm{b} /$ & 98.9 & 96.6 & 92 & 97.8 & 96.7 & 56 & 100.0 & 96.4 \\
\hline 5 & /j/ & 96.6 & 94.0 & 67 & 95.5 & 92.5 & 44 & 97.7 & 95.5 \\
\hline 6 & $/ \mathrm{n} /$ & 97.6 & 93.4 & 285 & 96.5 & 91.6 & 147 & 98.6 & 95.2 \\
\hline 7 & $/ \mathrm{d} /$ & 94.2 & 93.3 & 118 & 95.8 & 94.1 & 53 & 92.5 & 92.5 \\
\hline 8 & $/ \mathrm{m} /$ & 95.6 & 92.1 & 167 & 93.4 & 89.8 & 87 & 97.7 & 94.3 \\
\hline 9 & $|s|$ & 94.6 & 89.7 & 169 & 95.3 & 90.5 & 98 & 93.9 & 88.8 \\
\hline 10 & $/ \mathrm{tf} /$ & 88.8 & 88.8 & 26 & 84.6 & 84.6 & 14 & 92.9 & 92.9 \\
\hline 11 & $/ \mathrm{k} /$ & 90.2 & 87.5 & 125 & 92.0 & 88.0 & 69 & 88.4 & 87.0 \\
\hline 12 & $/ p /$ & 96.7 & 87.4 & 114 & 96.5 & 87.7 & 62 & 96.8 & 87.1 \\
\hline 13 & $/ \mathrm{g} /$ & 90.3 & 87.0 & 47 & 87.2 & 87.2 & 15 & 93.3 & 86.7 \\
\hline 14 & $\mid \mathrm{V} /$ & 82.8 & 82.8 & 14 & 92.9 & 92.9 & 11 & 72.7 & 72.7 \\
\hline 15 & /t/ & 91.4 & 79.6 & 223 & 89.2 & 78.9 & 157 & 93.6 & 80.3 \\
\hline 16 & /f/ & 78.6 & 78.6 & 21 & 90.5 & 90.5 & 9 & 66.7 & 66.7 \\
\hline 17 & $/ r /$ & 87.5 & 76.7 & 127 & 85.0 & 69.3 & 69 & 89.9 & 84.1 \\
\hline 18 & $/ \mathrm{h} /$ & 79.3 & 75.7 & 14 & 78.6 & 71.4 & 10 & 80.0 & 80.0 \\
\hline 19 & $|z|$ & 91.4 & 74.3 & 98 & 89.8 & 69.4 & 43 & 93.0 & 79.1 \\
\hline 20 & $|\theta|$ & 78.6 & 71.5 & 7 & 57.1 & 42.9 & 5 & 100.0 & 100.0 \\
\hline 21 & $|x|$ & 75.9 & 69.2 & 20 & 60.0 & 55.0 & 12 & 91.7 & 83.3 \\
\hline 22 & /I/ & 82.3 & 67.2 & 64 & 73.4 & 50.0 & 90 & 91.1 & 84.4 \\
\hline 23 & /ð/ & 66.7 & 66.7 & 41 & 63.4 & 63.4 & 10 & 70.0 & 70.0 \\
\hline 24 & /S/ & 66.7 & 66.7 & 3 & 33.3 & 33.3 & 1 & 100.0 & 100.0 \\
\hline 25 & $|x|$ & 83.4 & 61.2 & 11 & 66.7 & 55.6 & 3 & 100.0 & 66.7 \\
\hline & $M$ & 88.3 & 83.1 & 84.2 & 84.5 & 78.8 & 47.5 & 92.0 & 87.3 \\
\hline & SD & 9.9 & 11.7 & 74.4 & 16.7 & 19.0 & 44.1 & 9.7 & 10.6 \\
\hline
\end{tabular}

\section{Rank Ordered Agreement by Target Manner Feature}

\begin{tabular}{|c|c|c|c|c|c|c|c|c|c|}
\hline \multirow[t]{2}{*}{ Rank } & \multirow[t]{2}{*}{$\begin{array}{l}\text { Target } \\
\text { Class }\end{array}$} & \multicolumn{2}{|c|}{$\begin{array}{l}\text { Mean of Both } \\
\text { Transcribers }\end{array}$} & \multicolumn{3}{|c|}{ Transcriber 1 (eight samples) } & \multicolumn{3}{|c|}{ Transcriber 2 (four samples) } \\
\hline & & Broad & Narrow & $\mathrm{n}$ & Broad & Narrow & $n$ & Broad & Narrow \\
\hline 1 & glide & 98.6 & 96.7 & 180 & 98.3 & 95.6 & 92 & 98.9 & 97.8 \\
\hline 2 & nasal & 96.2 & 92.3 & 466 & 94.8 & 90.3 & 244 & 97.5 & 94.3 \\
\hline 3 & affricate & 90.0 & 90.0 & 29 & 86.2 & 86.2 & 16 & 93.8 & 93.8 \\
\hline 4 & stop & 93.4 & 86.8 & 719 & 92.9 & 87.2 & 412 & 93.9 & 86.4 \\
\hline 5 & fricative & 92.0 & 86.7 & 489 & 91.2 & 84.9 & 249 & 92.8 & 88.4 \\
\hline \multirow[t]{3}{*}{6} & liquid & 85.9 & 73.6 & 191 & 81.2 & 62.8 & 159 & 90.6 & 84.3 \\
\hline & $M$ & 92.7 & 87.7 & 345.7 & 90.8 & 84.5 & 195.3 & 94.6 & 90.8 \\
\hline & SD & 4.5 & 7.8 & 255.4 & 6.2 & 11.3 & 138.8 & 3.1 & 5.2 \\
\hline
\end{tabular}

a Excluding the infrequent $/ 3 /$ but including the two rhotic vowels $/ 3 /$ and $/ x /$

220 diacritics when transcribing with the analog system and 203 with the digital system (an 8\% decrease), averaging approximately 55 and 51 diacritics per sample, respectively. For the same samples, Transcriber 2 used 291 diacritics with the analog system and 332 with the digital system (a 14\% increase), averaging approximately 73 and 83 diacritics, respectively, per sample. 
Second, as shown in Table V, the greatest agreement discrepancies between broad and narrow transcription occurred on target sounds that have a variety of error and non-error allophones. For example, the large differences in the broad and narrow agreement percentages for the /z/ phoneme, and for fricatives as a manner class, are associated with the many error (e.g., dentalized, lateralized) and non-error (e.g., palatalized, retroflexed) allophones produced by children with speech sound disorders (cf. Shriberg, 1993: Appendix). The relatively large differences in the broad versus narrow agreement percentages for / $t$ / were associated with narrow transcription of the several non-error allophonic variations of /t/ (e.g., aspirated/unaspirated, released/unreleased, frictionalized).

A third source of support indicating that diacritic use was the primary source of disagreements in both systems concerns the rank-ordering of analog/digital agreement as listed in Table V. The order of entries in the Target Sound column is highly concordant with the order of consonant acquisition in both typical and atypical speakers. That is, later acquired sounds are more often in error, including distortion error-types that require diacritic-level (i.e., narrow) transcription to describe. Rather than per-sound or per-feature concordance, however, the order of entries in the Target Sound column is consistent with the division of speech acquisition into three developmental sound classes termed the Early-8 (/m/, /b/, /j/, /n/, /w/, /d/, /p/, /h/), Middle-8 (/t/, /g/, /k/, /g/, /f/, /v/, /tf/, /dz/), and Late-8 (/S/, / $\theta /, / \mathrm{s} /, / \mathrm{z} /, / \circlearrowright /, / 1 /, / \mathrm{r} /, / 3 /$ ) sounds (Shriberg, 1993). Validity data for the construct of similar developmental classes in children with both typical speech and speech delay of unknown origin indicate that the eight sounds of each class are acquired before all of the sounds of each later developing class (Shriberg, 1993). As shown in table 5, the order of sounds based on transcription agreement using analog and digital systems is generally concordant (concordance $=87.5 \%$ ) with the eight sounds listed above for each of the three developmental sound classes. The only three exceptions (excluding the two rhotic vowels) for the 24 consonants are /dz/ (which is based on only a few tokens), /y/, and /s/. Notice also that the ordering of agreement by manner features at the bottom of Table $\mathrm{V}$ generally follows the developmental order of speech acquisition.

To summarize, these quantitative agreement analyses indicated that transcribers obtained the same levels of interjudge agreement using analog versus digital audio systems that investigators have reported for transcription agreement within and between analog systems. Until estimates can be obtained from larger-scale studies in which many samples are transcribed at least twice by many transcribers using a variety of digital compared to analog systems, the present data do not allow conclusions about the likelihood of enhanced transcriber reliability using digital systems. However, the last section of this report provides some qualitative information supporting this possibility.

\section{Prosody-voice coding}

Following standard procedures for the screening instrument used in this study, prosodyvoice data were obtained from the first 24 utterances in the sample that were classified as eligible for prosody-voice coding (Shriberg et al., 1990). As noted previously, glossing a conversational speech sample for the purpose of prosody-voice coding includes a set of procedures to exclude utterances that are not appropriate to code due to technical, behavioral, or linguistic factors (e.g., microphone distance, examiner overtalk). For two of the eight participants, this resulted in only 16 utterances available for prosody-voice coding. It is important to note that although the speech samples in the present study purposely over-sampled more severely involved children, utterances with inappropriate pros- 
ody are characteristically infrequent in children with speech delay of unknown origin, at all levels of severity of speech sound involvement (Shriberg \& Kwiatkowski, 1994).

Completion time analyses. Table II includes the completion time data for prosody-voice coding of the eight or four samples completed by each transcriber using the two audio systems. Average prosody-voice coding times for the second compared to the first listening series for Transcriber 1 (15.0 $\mathrm{min}, 16.3 \mathrm{~min}$, respectively) and Transcriber 2 (34.5 min, $39.3 \mathrm{~min}$ ) were shorter, with small (0.27) and medium (0.64) ESs associated respectively with these differences. As found for glossing and phonetic transcription completion times, Transcriber 2 averaged over twice as long to complete prosody-voice coding of the subset of four speech samples as Transcriber 1. As tabulated by audio system, both transcribers took less time to complete prosody-voice coding with the digital compared to the analog system, although neither of the ESs was statistically significant. Transcriber 1 averaged 16.0 min using the analog system and 15.3 min using the digital system ( $\mathrm{ES}=0.15$; negligible). Transcriber 2 averaged $40.5 \mathrm{~min}$ using the analog system and 33.3 min using the digital system ( $\mathrm{ES}=1.10$; very large). As shown in the individual sample comparisons in Table II, Transcriber 2 appeared to have substantially reduced prosody-voice coding times for at least one of the four speech samples using the digital (36 min) compared to the analog (51 min) system.

Between-system agreement. Table VI is a summary of the point-to-point prosody-voice agreement data obtained for each transcriber. Once again, these comparisons estimate the intrajudge agreement for transcribers coding participants' prosody-voice characteristics using the digital compared to the analog system. Because inappropriate prosodyvoice behaviors occur relatively infrequently in children with speech delay (with the exception of the frequent vocal roughness coded as inappropriate laryngeal quality), the second and third rows in Table VI provide the number of utterances coded as inappropriate for each variable using the two audio systems. For this reason as well, ES estimates on the between-system agreement were deemed inappropriate.

The agreement percentages in the bottom row of Table VI indicate generally high rates of agreement using the digital compared to the analog system. As noted previously, however, these percentages are heavily weighted by the low occurrence of utterances with inap-

Table VI. Number of inappropriate prosody-voice codes perceived by transcribers using the analog and digital audio systems.

\begin{tabular}{|c|c|c|c|c|c|c|c|c|c|c|c|c|c|c|}
\hline & \multicolumn{6}{|c|}{ Prosody } & \multicolumn{8}{|c|}{ Voice } \\
\hline & \multicolumn{2}{|c|}{ Phrasing } & \multicolumn{2}{|c|}{ Rate } & \multicolumn{2}{|c|}{ Stress } & \multicolumn{2}{|c|}{ Loudness } & \multicolumn{2}{|c|}{ Pitch } & \multicolumn{2}{|c|}{$\begin{array}{l}\text { Laryngeal } \\
\text { Quality }\end{array}$} & \multicolumn{2}{|c|}{ Resonance } \\
\hline & $\operatorname{Tr} 1^{a}$ & $\operatorname{Tr} 2$ & $\operatorname{Tr} 1$ & $\operatorname{Tr} 2$ & $\operatorname{Tr} 1$ & $\operatorname{Tr} 2$ & $\operatorname{Tr} 1$ & $\operatorname{Tr} 2$ & $\operatorname{Tr} 1$ & $\operatorname{Tr} 2$ & $\operatorname{Tr} 1$ & $\operatorname{Tr} 2$ & $\operatorname{Tr} 1$ & $\operatorname{Tr} 2$ \\
\hline $\begin{array}{l}\text { Total } \\
\text { Utterances } \\
\text { Coded }\end{array}$ & 184 & 88 & 184 & 88 & 184 & 88 & 184 & 88 & 183 & 88 & 183 & 88 & 184 & 88 \\
\hline \multicolumn{15}{|c|}{ Inappropriate Codes } \\
\hline Analog & 26 & 14 & 7 & 5 & 20 & 21 & 7 & 7 & 4 & 2 & 40 & 5 & 21 & 4 \\
\hline Digital & 25 & 13 & 6 & 3 & 9 & 22 & 7 & 9 & 4 & 2 & 48 & 6 & 8 & 6 \\
\hline Agreement (\%) & 99.5 & 98.9 & 98.4 & 97.7 & 92.4 & 80.7 & 97.8 & 97.7 & 98.9 & 100.0 & 86.9 & 96.6 & 89.7 & 97.7 \\
\hline
\end{tabular}

a $\operatorname{Tr} 1$ = Transcriber $1 ; \operatorname{Tr} 2=\operatorname{Transcriber} 2$ 
propriate prosody-voice. A more sensitive comparison of possible analog/digital differences is provided in the middle two rows in Table VI, which include the number of utterances coded as inappropriate for each variable using the two audio systems. The intrajudge data are fairly similar for most comparisons, excepting Transcriber 1's analog/ digital data for Stress and Resonance. For both variables, Transcriber 1 coded fewer utterances as inappropriate using the digital system. Discussion of these findings is deferred to the last section, which provides qualitative data on the transcribers' observations and preferences.

\section{Validity}

A series of 24 speech and prosody-voice profile outputs from the software suite (Shriberg et al., 2001) provided statistical comparisons of findings based on transcription and prosody-voice coding data, using the digital compared to the analog audio system. These comparisons were viewed as concurrent validity estimates, because they tested whether children's speech and prosody-voice scores based on the digital system differed from their severity scores based on the analog system (i.e., as indicated by the magnitude of effect sizes). The speech profiles included comparisons of targets cross-tabulated by several types of linguistic units, including (a) class (sonorants, obstruents; consonants, vowels/diphthongs), (b) feature (place, manner, voicing), (c) context (singletons, clusters, position in word), (d) phoneme (individual sounds), (e) speech error type (omission, substitution, distortion), (f) natural process (e.g., final consonant deletion), and (g) error (e.g., dentalized sibilants) and non-error (e.g., palatalized sibilants) allophone. The prosody-voice profiles, as described previously, included scores in seven suprasegmental domains and subtypes within each domain.

Table VII is a summary of representative findings from these comparisons based on transcripts generated with the analog versus digital audio system. For each of the two transcribers, descriptive and inferential statistics are provided for analog/digital comparisons on eight speech severity/typology metrics, their subscales, and seven prosodyvoice domains. These metrics have been described elsewhere (Shriberg, Austin, Lewis, McSweeny, \& Wilson, 1997a, b). Essentially, scores on five of the first eight measures and their subscales (PCC, PCI, PVC, PPC, II), as well as the seven prosody-voice subscales, indicate the percentage of correct or appropriate responses in each domain. Scores on the three error type metrics (POE, PSE, PDE) indicate the percentage of incorrect responses associated with each type of error (omission, substitution, distortion).

Overall, the descriptive and inferential statistical data in Table VII support the concurrent validity of scores obtained with the digital system compared to the analog system. First, the means for the digitally-based compared to the analog-based severity and error-type indices were generally within a few percentage points of each other and within the standard error of measurement for these metrics, as reported elsewhere (Shriberg et al., 1997a). Second, the effect sizes associated with these descriptive data ranged from negligible to medium, with none reaching the level of large or greater. Of the 36 severity comparisons in Table VII (i.e., excluding the three error type comparisons for each transcriber), there was, however, a trend for lower speech competency scores obtained with the digital audio system (i.e., indicating less competence) than those obtained with the analog system. Specifically, 25 of 36 comparisons (69\%) were lower. Third, with multiple comparisons using a liberal parametric statistic to test for significant differences on as few as four sample comparisons (comparable to a $t$ test), none of the Hedges' corrected 95\% confidence interval tests were significant at the .05 
Table VII. Summary of the validity findings for speech and prosody-voice data obtained using analog versus digital audio systems.

\begin{tabular}{|c|c|c|c|c|c|c|c|c|c|c|c|c|c|c|}
\hline \multirow[b]{3}{*}{ Variable } & \multicolumn{7}{|c|}{ Transcriber 1} & \multicolumn{7}{|c|}{ Transcriber 2} \\
\hline & \multicolumn{2}{|c|}{ Analog } & \multicolumn{2}{|c|}{ Digital } & \multicolumn{3}{|c|}{ Comparison } & \multicolumn{2}{|c|}{ Analog } & \multicolumn{2}{|c|}{ Digital } & \multicolumn{3}{|c|}{ Comparison } \\
\hline & $M$ & SD & M & SD & $\mathrm{ES}^{\mathrm{a}}$ & Desc. $^{b}$ & $p^{c}$ & $M$ & SD & $M$ & $\mathrm{SD}$ & ES & Desc. & $p$ \\
\hline \multicolumn{15}{|c|}{$\begin{array}{l}\text { Consonants } \\
\text { PCC }\end{array}$} \\
\hline Early-8 & 84.1 & 8.6 & 82.7 & 9.4 & 0.14 & $\mathrm{~N}$ & .77 & 88.7 & 7.3 & 87.6 & 7.8 & 0.13 & $\mathrm{~N}$ & .84 \\
\hline Middle-8 & 61.4 & 23.1 & 58.6 & 25.4 & 0.11 & $\mathrm{~N}$ & .82 & 71.2 & 11.2 & 64.8 & 9.9 & 0.53 & $M$ & .42 \\
\hline Late-8 & 35.5 & 19.2 & 33.5 & 19.7 & 0.10 & $\mathrm{~N}$ & .84 & 43.1 & 8.2 & 38.5 & 9.6 & 0.46 & $\mathrm{~S}$ & .49 \\
\hline Total & 63.5 & 13.6 & 61.3 & 14.5 & 0.15 & $\mathrm{~N}$ & .76 & 70.3 & 7.8 & 66.8 & 8.0 & 0.39 & $\mathrm{~S}$ & .55 \\
\hline \multicolumn{15}{|l|}{$\mathrm{PCl}$} \\
\hline Early-8 & 97.7 & 4.7 & 97.7 & 4.7 & 0.00 & $\mathrm{~N}$ & 1.00 & 98.4 & 3.1 & 96.9 & 6.3 & 0.27 & $\mathrm{~S}$ & .67 \\
\hline Middle-8 & 77.3 & 21.2 & 73.6 & 26.2 & 0.15 & $\mathrm{~N}$ & .76 & 76.7 & 25.8 & 71.7 & 22.8 & 0.18 & $\mathrm{~N}$ & .78 \\
\hline Late-8 & 79.1 & 14.0 & 68.5 & 18.1 & 0.62 & $M$ & .21 & 85.4 & 8.9 & 81.4 & 9.2 & 0.38 & $\mathrm{~S}$ & .56 \\
\hline Total & 85.6 & 10.5 & 81.1 & 13.5 & 0.35 & $\mathrm{~S}$ & .47 & 87.6 & 8.8 & 84.1 & 11.1 & 0.30 & $\mathrm{~S}$ & .64 \\
\hline POE & 14.2 & 8.4 & 14.6 & 8.9 & 0.04 & $\mathrm{~N}$ & .93 & 6.6 & 4.1 & 8.5 & 5.2 & 0.35 & $\mathrm{~S}$ & .59 \\
\hline PSE & 17.2 & 7.3 & 19.1 & 7.0 & 0.25 & $\mathrm{~S}$ & .60 & 14.5 & 5.8 & 16.3 & 4.8 & 0.29 & $\mathrm{~S}$ & .66 \\
\hline PDE & 5.1 & 1.5 & 5.0 & 1.5 & 0.07 & $\mathrm{~N}$ & .89 & 8.6 & 1.7 & 8.4 & 1.2 & 0.08 & $\mathrm{~N}$ & .90 \\
\hline \multicolumn{15}{|c|}{ Vowels/Diphthongs } \\
\hline PVC & 93.3 & 2.2 & 92.1 & 2.5 & 0.50 & M & .31 & 93.5 & 2.8 & 92.9 & 2.0 & 0.19 & $\mathrm{~N}$ & .77 \\
\hline \multicolumn{15}{|c|}{ Phonemes andWords } \\
\hline PPC & 75.5 & 8.6 & 73.6 & 9.4 & 0.19 & $\mathrm{~N}$ & .69 & 79.5 & 5.2 & 77.2 & 5.2 & 0.39 & $\mathrm{~S}$ & .55 \\
\hline II & 84.9 & 13.2 & 86.2 & 10.5 & 0.10 & $\mathrm{~N}$ & .84 & 87.5 & 14.3 & 89.2 & 10.3 & 0.12 & $\mathrm{~N}$ & .85 \\
\hline \multicolumn{15}{|l|}{ PVSP } \\
\hline Phrasing & 85.9 & 5.4 & 86.5 & 6.2 & 0.08 & $\mathrm{~N}$ & .86 & 84.4 & 4.0 & 85.4 & 5.4 & 0.19 & $\mathrm{~N}$ & .77 \\
\hline Rate & 96.1 & 4.8 & 96.9 & 3.7 & 0.17 & $\mathrm{~N}$ & .72 & 93.8 & 5.4 & 96.4 & 2.6 & 0.53 & M & .42 \\
\hline Stress & 88.8 & 5.8 & 86.5 & 9.1 & 0.29 & $\mathrm{~S}$ & .55 & 77.1 & 15.8 & 74.0 & 14.2 & 0.18 & $\mathrm{~N}$ & .78 \\
\hline Loudness & 96.4 & 5.7 & 96.4 & 4.7 & 0.00 & $\mathrm{~N}$ & 1.00 & 92.2 & 6.0 & 89.6 & 5.4 & 0.40 & $\mathrm{~S}$ & .54 \\
\hline Pitch & 97.9 & 3.3 & 97.9 & 3.9 & 0.01 & $\mathrm{~N}$ & .98 & 97.9 & 4.2 & 97.9 & 4.2 & 0.00 & $\mathrm{~N}$ & 1.00 \\
\hline Laryngeal & 78.4 & 15.7 & 73.9 & 22.3 & 0.22 & $\mathrm{~S}$ & .65 & 94.8 & 6.3 & 93.8 & 7.2 & 0.13 & $\mathrm{~N}$ & .83 \\
\hline Resonance & 87.2 & 17.2 & 95.3 & 5.7 & 0.60 & M & .23 & 94.3 & 8.9 & 91.7 & 11.8 & 0.22 & $\mathrm{~S}$ & .74 \\
\hline
\end{tabular}

a $\mathrm{ES}=$ Effect Size (Cohen's $d$-Hedges corrected; Cohen 1988).

${ }^{\mathrm{b}}$ Desc. = Descriptor $(\mathrm{N}=$ Negligible, $\mathrm{S}=$ Small, $\mathrm{M}=$ Medium, $\mathrm{L}=$ Large, $\mathrm{V}=$ Very Large, $\mathrm{E}=$ Extremely Large; see text for key for these descriptors).

c All values were non-significant.

level or beyond. Finally, comparisons at the diacritic level (e.g., dentalized, lateralized, palatalized sibilants) not shown in Table VII also indicated that there were no notable differences in scores assigned to children based on transcriptions obtained using the digital system compared to the analog audio system.

Figure 1 provides an additional validity perspective, consistent with the trend noted previously for participants to receive lower competency scores for transcription based on data obtained with the digital system compared to the analog audio system. The top panel includes findings for Transcriber 1 and the bottom panel for Transcriber 2. The numeric and graphic sections of each panel aggregate productions of all consonant sounds in the transcripts by class (sonorant, obstruent), voice (voiced, voiceless), and manner feature (nasal, glide, stop, fricative, affricate, liquid). These data indicate that average participant scores based on transcription from the digital system (square symbol) were lower across nearly all comparisons than those obtained with the analog system (circle symbol). This trend was more notable for Transcriber 2 , with generally small (one medium) effect sizes. 

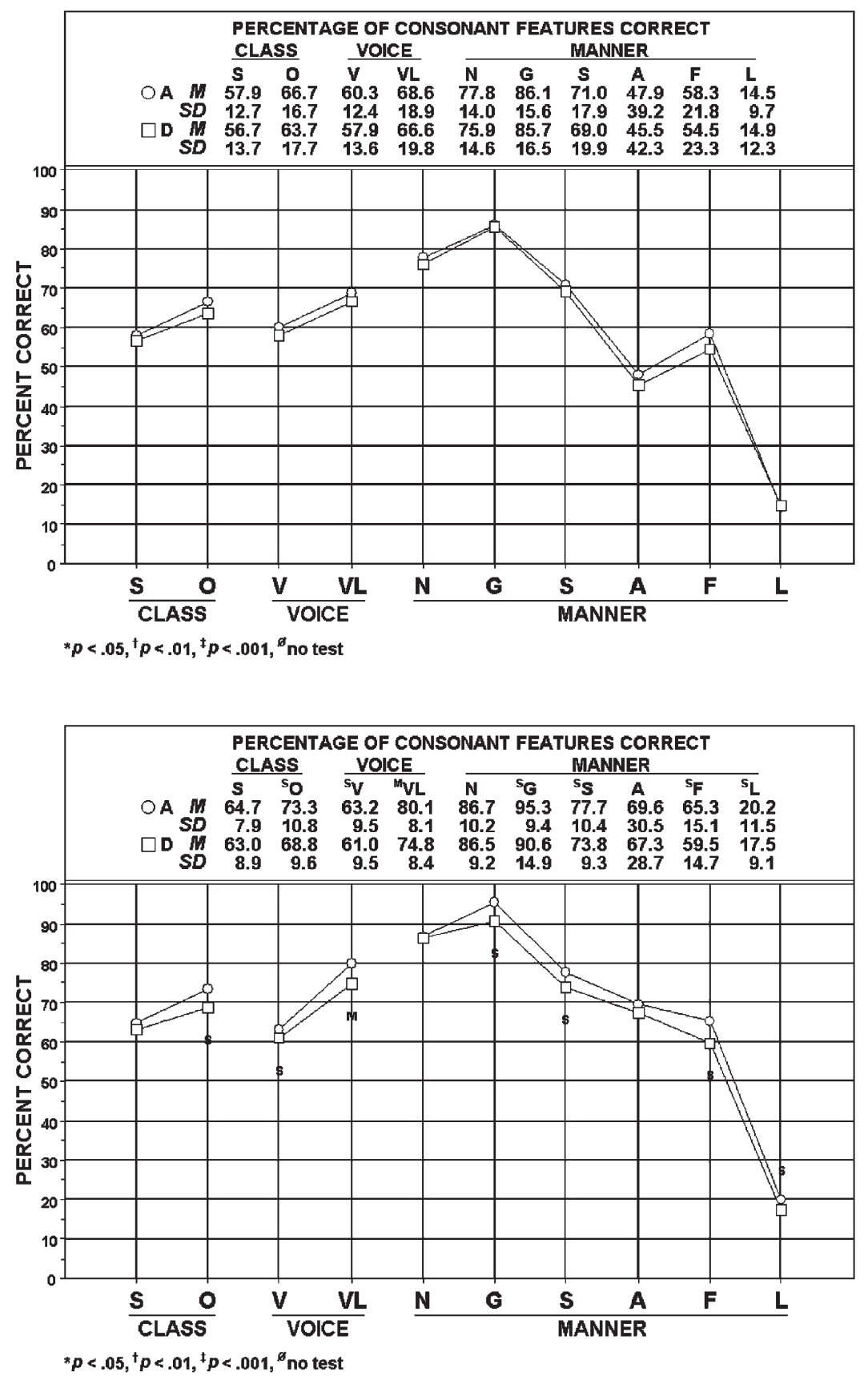

Figure 1. Sample validity findings for transcription outcomes based on data obtained with the digital (square) and analog (circle) audio systems for Transcriber 1 (top panel) and Transcriber 2 (bottom panel).

\section{Transcribers' observations on signal quality and operational efficiencies}

In addition to the quantitative data just reviewed, the research design included qualitative reports on transcribers' perceptions of the signal qualities and operational efficiencies of the digital system compared to the audio system. Table VIII is a summary 
Table VIII. Transcribers' anecdotal observations on the strengths and weaknesses of the two audio systems.

Observations on Signal Quality Analog Digital

Could hear some things more clearly on digital system but would need to listen to the same sample on each system side by side to confirm this impression

When the original audio was "muffled," quiet, or not clear, it was degraded on both the analog and digital systems. Still, I was able to hear some things more clearly in the digital system.

I felt more confident transcribing using the digital system because the sound quality was generally better than that from the analog system. The signal seemed less distorted, and I was better able to hear fricative distortions, voicing changes, and the presence (or absence) of word final consonants, which are typically brief and weak.

There seemed to be fewer times when I used the "benefit of the doubt" a rule using the digital system. For example, I was more likely to perceive a dentalized /s/ without second guessing myself.

+
+
+
+
+

Observations on Operational Features

The tapes had to be rewound, fast-forwarded, and played at regular speeds to find the conversational speech sample among the other speech tasks in the assessment protocol.

One tape was "eaten" by the analog system and it took me 20 minutes to repair the tape. After repair, the tape was still a little wrinkled and frayed, and that section did not play well.

The disc drive was sometimes noisy (humming, rattling) when the disc was spinning in the drive. But once loaded, the disc could be removed from the drive so that this noise was not a problem during glossing, transcription, and coding.

I felt less confident judging appropriate resonance using the digital system compared to the analog system.

It took anywhere from 30 seconds to almost 2 minutes to open a .wav file in the digital system.

a The "benefit of the doubt" convention is used when a transcriber is in doubt about the transcription. The convention is to transcribe all such occurrences as correct.

of their comments, with plus ("+") and minus ("-") signs used to indicate a perceived strength or limitation of each system, with the signs for qualified or conditional observations placed within parentheses.

Based on a simple tally of the number and type of observations for each system, transcribers appeared to favor the digital system for glossing, transcription, and prosodyvoice coding. In comparison to the analog system, the digital system was perceived as having a higher quality signal and more efficient operational features. It is important to note another efficiency feature associated with the digital system that transcribers did not comment on until after the study. Transcribers reported that, in comparison to the analog system, the digital system allowed them to find the speech sample much more easily and rapidly among other recorded tasks in the assessment protocol.

\section{Summary and conclusions}

Several methodological constraints limiting generalizations from this small-scale study have been noted in the subsection discussions. Primary needs to address in larger studies include (a) greater number of transcribers reflecting different levels of training, (b) greater numbers and variability of speech samples, including those from speakers with more severe prosody-voice involvement, (c) increased time intervals between listening 
series, and (d) increased comparisons among alternative types of analog and digital recording and playback systems (e.g., monaural versus stereo configurations, alternative microphones, alternative earphones). Until data from such cross-validation and extension studies become available, we suggest that the findings from the present study support the following conclusions and recommendations.

First, as indicated in Table VIII, both transcribers appeared to prefer digital playback systems of the type described in this report to the type of analog playback device used for the past several decades in the clinic and laboratory. At least for speech signals, digital systems appear to have better signal fidelity (currently, there is heated debate on analog/ digital sound quality issues among music aficionados) as well as many operational efficiencies. A study series currently in progress in our laboratory is assessing additional variables, using a laptop system for both audio playback and on-line transcript entry and transcript analysis. For readers interested in more information on this topic, Chial (2003) has assembled a technical report that includes guidelines on transitioning to digital technology for recording, storing, and playback of speech tasks for the purposes of perceptual and acoustic analyses. This technical report can be downloaded at http://www.waisman.wisc.edu/phonology/TREP13.PDF.

Second, the data from this study suggest that there may be some differences associated with transcription using digital systems that might affect standardization. Although the concurrent validity data suggested that group-level outcomes were generally not significantly different, the signal quality differences cited by the transcribers and several of the glossing and reliability findings suggest the need for caution at the level of individual samples when interpreting clinical and research data obtained with digital compared to extant analog audio recording and playback systems. Until larger-scale studies identify and quantify potentially important differences associated with each system, including per-speaker contrasts in addition to grouped data, it would seem prudent to include control groups assessed using digital systems in every study requiring a critical comparison of digital samples to reference data. In clinical and research contexts, speech-language pathologists could assess possibly relevant local speech differences (e.g., dialectal) using analog/digital comparisons similar to those used in the present study. Optimally sensitive studies might compare findings from the most difficult tokens presented to listeners in live voice, analog, and digital signal conditions.

Finally, as concluded in most methodological studies of phonetic transcription and prosody-voice coding, auditory-perceptual approaches appear to have limits on reliability that may be unacceptable for certain clinical and research needs. Although not directly tested in the present study, even high quality digital recording and playback systems lack the information on speech events that is needed to maximize the validity and reliability of narrow phonetic transcription. As in other areas of communicative disorders, transcription systems that include simultaneous displays of signal properties (e.g., wave forms, spectrograms) provide substantial assistance in identifying specific speech errors and differences. Such acoustic-aided systems are currently available, although transcripts derived from these instruments also require comparative research study relative to standardization issues.

\section{Acknowledgments}

We thank the following research assistants in Pittsburgh and Madison for their important contributions to this study: Mitzi Ahn, Denise Balason, Jill Brady, Stacey Carr, Cath- 
erine Coffey, Stephanie Nixon, and Alison Scheer. Special thanks to Christie Tilkens for her assistance with transcription and prosody-voice coding. This research was supported by collaborative grants from the National Institute on Deafness and Other Communicative Disorders, NIDCD DC00496 and NIDCD DC00822.

\section{References}

Bamattre, R. (1995). How two small hospitals moved from analog to digital transcription. Journal of the American Health Information Management Association, 66, 48-49.

Beukelman, D. (2000). Recording, refining, transferring, and storing audio samples using Apple Macintosh computer technology; accessed February 22, 2005, http://aac-rerc.com/archive_aac-rerc/pages/ wtpapers.html

Bunta, F., Ingram, K., \& Ingram, D. (2003). Bridging the digital divide: Aspects of computerized data collection and analysis for language professionals. Clinical Linguistics and Phonetics, 17, 217-240.

Chial, M. R. (2003). Suggestions for computer-based audio recording of speech samples for perceptual and acoustic analyses (Tech. Rep. No. 13). Phonology Project, Waisman Center, University of Wisconsin- Madison; accessed February 22, 2005; http://www.waisman.wisc.edu/phonology/TREP13.PDF

Cohen, J. (1988). Statistical Power Analysis for the Behavioral Sciences. Hillsdale, NJ: Lawrence Erlbaum.

Doherty, E. T., \& Shipp, T. (1988). Tape recorder effects on jitter and shimmer extraction. Journal of Speech and Hearing Research, 31, 485-490.

Evans, J. L., \& Miller, J. (1999). Language sample analysis in the 21st century. Seminars in Speech and Language, 20, 101-116.

Farinella, K., DeLeo, J., Metz, D. E., Schiavetti, N., \& Whitehead, R. L. (1998). Influence of analog versus digital recordings on speech naturalness judgments. Contemporary Issues in Communication Science and Disorders, 25, 49-53.

Hedges, L., \& Olkin, I. (1985). Statistical Methods for Meta-Analysis (pp. 75-106). New York: Academic Press.

Hopkins, W. G. (2003). A new view of statistics; accessed February 22, 2005; http://www.sportsci.org/resource/stats/effect.html

Ladefoged, P. (2003). Phonetic Data Analysis: An Introduction to Fieldwork and Instrumental Techniques. Malden, MA: Blackwell.

Long, S. H. (1999). Technology applications in the assessment of children's language. Seminars in Speech and Language, 20, 117-132.

MacWhinney, B. (2005). Talkbank: Digital Audio Guide; accessed February 22, 2005; http://talkbank. org/da/

McSweeny, J. L., \& Shriberg, L. D. (1995). Segmental and suprasegmental transcription reliability (Tech. Rep. No. 2). Phonology Project, Waisman Center, University of Wisconsin-Madison; accessed February 22, 2005; http://www.waisman.wisc.edu/phonology/TREP02.PDF

Perry, C. K., Ingrisano, D. R. S., \& Blair, W. B. (1996). The influence of recording systems on jitter and shimmer estimates. American Journal of Speech-Language Pathology, 5, 86-90.

Plichta, B. (2002). Best practices in the acquisition, processing and analysis of acoustic speech signals. Eleventh International Conference on Methods in Dialectology, Joensuu, Finland; accessed February 22, 2005; http://www.historicalvoices.org/flint/extras/Audio-technology.pdf

Shriberg, L. D. (1986). PEPPER: Programs to Examine Phonetic and Phonologic Evaluation Records. Hillsdale, NJ: Lawrence Erlbaum.

Shriberg, L. D. (1993). Four new speech and prosody-voice measures for genetics research and other studies in developmental phonological disorders. Journal of Speech and Hearing Research, 36, 105-140.

Shriberg, L. D., Allen, C. T., McSweeny, J. L., \& Wilson, D. L. (2001). PEPPER: Programs to Examine Phonetic and Phonologic Evaluation Records, computer software. Madison, WI: Waisman Center Research Computing Facility, University of Wisconsin-Madison.

Shriberg, L. D., Austin, D., Lewis, B. A., McSweeny, J. L., \& Wilson, D. L. (1997a). The Percentage of Consonants Correct (PCC) metric: Extensions and reliability data. Journal of Speech, Language, and Hearing Research, 40, 708-722. 
Shriberg, L. D., Austin, D., Lewis, B. A., McSweeny, J. L., \& Wilson, D. L. (1997b). The Speech Disorders Classification System (SDCS): Extensions and lifespan reference data. Journal of Speech, Language, and Hearing Research, 40, 723-740.

Shriberg, L. D., \& Kent, R. D. (2003). Clinical Phonetics, third edition. Boston, MA: Allyn \& Bacon.

Shriberg, L. D., \& Kwiatkowski, J. (1980). Natural Process Analysis: A Procedure for Phonological Analysis of Continuous Speech Samples. New York: Macmillan.

Shriberg, L. D., \& Kwiatkowski, J. (1994). Developmental phonological disorders I: A clinical profile. Journal of Speech and Hearing Research, 37, 1100-1126.

Shriberg, L. D., Kwiatkowski, J., \& Rasmussen, C. (1990). The Prosody-Voice Screening Profile. Tucson, AZ: Communication Skill Builders.

Shriberg, L. D., \& Lof, G. L. (1991). Reliability studies in broad and narrow phonetic transcription. Clinical Linguistics and Phonetics, 5, 225-279.

Titze, I. R., Horii, Y., \& Scherer, R. C. (1987). Some technical considerations in voice perturbation measurements. Journal of Speech and Hearing Research, 30, 252-260.

Weston, A. D., \& Shriberg, L. D. (1992). Contextual and linguistic correlates of intelligibility in children with developmental phonological disorders. Journal of Speech and Hearing Research, 35, 1316-1332.

Wolfe, V., Martin, D., Borton, T., \& Youngblood, H. C. (2003). The effect of clinical experience on cue trading for the /r-w/ contrast. American Journal of Speech-Language Pathology, 12, 221-228.

Zafar, A., Overhage, J. M., \& McDonald, C. J. (1999). Continuous speech recognition for clinicians. Journal of the American Medical Informatics Association, 6, 195-204.

Appendix: Description of the recording and playback devices used in this study.

\section{Recording}

Conversational speech samples were recorded using a Shure omnidirectional UHF Wireless microphone system (Model UI-UA), with mouth-to-microphone distance monitored at approximately 6 inches. The audio signal was routed from the body-pack transmitter to the dual channel diversity receiver of the Shure system and to a Mackie 1202-VLZ Pro 12-channel mixer. Signal amplitudes were adjusted at the beginning of the session for maximum gain without distortion and were not subsequently changed. The output from the Mackie mixer fed a TEAC GX-1 integrated recorder. The TEAC was used to digitally record the conversational speech samples (.dat files) on a Sony SDX1-25C 170m Advanced Intelligent Tape (AIT) recorder.

Of the eight conversational samples used in the present study, three were digitized at a sampling rate of $20 \mathrm{kHz}$ and five at $50 \mathrm{kHz}$, both with 16 bits of amplitude resolution (quantization factor). The monaural acoustic waveform was monitored using a Dataq Instruments DI-700 USB data acquisition system and WinDaq/Lite waveform acquisition software. The dat files were subsequently converted to .wav files using GX View software. The eight files for the present study were then copied onto a CD-R using Prassi Primo DVD 2.0 software. For the present study, the acoustic signal was also transmitted from the systems described above to a Marantz PMD 502 stereo cassette recorder, where it was recorded on Maxell Professional Communicator Series C60Low Noise/High Output analog cassette tapes. Prior to each recording, the signal on the Marantz recorder was adjusted for optimum gain without distortion and not subsequently changed.

\section{Playback}

Analog system. The analog playback system was a Dictaphone Thought Master 2250 transcriber, one of several 280 and 2250 machines in the Thought Master series used by the Madison group in prior reported studies of child speech sound disorders. Technical specifications listed in the manual for these rugged playback devices include a frequency response of $200 \mathrm{~Hz}$ to $5000 \mathrm{~Hz} \pm 3 \mathrm{~dB}$, a signal to noise ratio of $40 \mathrm{~dB}$, wow and flutter of .5\%, and a harmonic distortion of $3.0 \%$ maximum at $400 \mathrm{~Hz}$ at the rated power output of 750 milliwatts. The length of playbacks was adjust- 
able; and start/stop, replays, and fast forward/rewind functions were controlled either by function levers or buttons on the console or by a foot pedal.

Digital system. The digital playback system hardware included a 933MHz Intel Pentium III computer with 384 MB memory, two 80 GB disk drives running Windows 98 second edition, and a Creative Technology (Audigy 2, SB0240) sound card. The audio signal was played through a pair of Yamaha YST-M101W magnetically shielded stereo speakers (rated at 3 watts/speaker and a frequency response of $80 \mathrm{~Hz}$ to $20000 \mathrm{~Hz} \pm 3 \mathrm{~dB}$ ) positioned at approximately the same distance from the transcriber as the speaker in the Dictaphone machine.

The application used for playback was a locally developed, $\mathrm{C}++$ program that uses Windows routines operating within the PEPPER software environment (Shriberg et al., 2001). The system included an XKeys foot pedal from P.I. Engineering - http://www.xkeys.com/xkeys/xkfoot.php that provided the same stop/start, variable rewind, and fast forward/rewind capabilities as the Dictaphone analog playback device.

A technical note. It may be useful, for others transitioning to digital recording/playback devices, to describe a technical challenge experienced when configuring the playback system to accommodate the $50 \mathrm{kHz}$ sampling rate for some of the conversational samples. We initially tried to play these speech samples on the same computer system described above, using a Creative Technology Sound Blaster PCI 128 (CT4750) sound card. The playback was noticeably slower than the original speech and contained audible clicks. A series of diagnostic analyses indicated two problems. First, the PCI 128 sound card could not keep pace with the sampling rate. Switching to the Audigy 2 sound card resolved the problem, as confirmed by oscilloscopic inspection of test .wav files and perceptual evaluation. The test files consisted of sample square waves with 50 samples per cycle and sample rates ranging from $20 \mathrm{kHz}$ to $50 \mathrm{kHz}$. Second, the driver for the sound card did not work with the recommended driver (WDM) but did work with an alternative (VXD). The point of this note is not a concern with the specifics of this system, because hardware and software options are always evolving. Rather, as discussed in the technical report motivated by this study (Chial, 2003), the general point is to underscore the array of challenging technical issues that require careful attention when assembling digital-based audio recording and playback systems for clinical and research applications. 